\title{
Índice glucémico y carga glucémica en el fruto de la especie vegetal Opuntia ficus-indica
}

\author{
María de Jesús Ibarra-Salas', Hilda Irene Novelo-Huerta', Marcela Alejandra De León-Salas', \\ Mayra Elisa Sánchez-Murillo², María del Carmen Mata-Obregón ${ }^{1}$ y Aurora de Jesús Garza-Juárez \\ ${ }^{1}$ Facultad de Salud Pública y Nutrición, Universidad Autónoma de Nuevo León (UANL), Monterrey, N.L.; ${ }^{2}$ Facultad de Ciencias Biológicas, UANL, \\ San Nicolás de los Garza, N.L. México
}

\section{Resumen}

Existe evidencia que apoya la utilidad clínica de que el índice glucémico (IG) y la carga glucémica (CG) podrían ser importantes en la prevención de enfermedades crónicas. Objetivo: Determinar el IG y la CG del fruto de Opuntia ficus-indica (tuna). Métodos: Se realizó un estudio analítico, transversal, con 25 voluntarios sanos con valores normales de índice de masa corporal, glucosa, hemoglobina glucosilada, colesterol y triglicéridos séricos, que mediante consentimiento informado aceptaron participar. Con los datos antropométricos y bioquímicos se evaluó la homogeneidad de la población usando análisis de componente principal (PCA). Se proporcionó el equivalente a $50 \mathrm{~g}$ de hidratos de carbono de alimento de prueba (tuna) y $50 \mathrm{~g}$ de dextrosa como alimento estándar para medir la curva de glucosa. El IG se determinó calculando el área bajo la curva por el método de triangulación. La CG se reportó como el producto del IG por la carga de hidratos de carbono proporcionada. Resultados y conclusiones: El IG de la tuna fue de $48.01 \pm 17.4$, clasificado como bajo, mientras que la CG fue de $24.0 \pm 8.7$, clasificada como alta. El análisis quimiométrico por PCA demostró que, en la selección de la población normal para la determinación del IG, es importante considerar los valores de colesterol y triglicéridos en sangre.

PALABRAS CLAVE: Índice glucémico. Carga glucémica. Opuntia ficus-indica.

\begin{abstract}
There is evidence that support the clinical usage of glycemic index (GI) and glycemic load (GL) in the prevention of chronic disease. Objective: To determine the Gl and GL of the Opuntia ficus-indica fruit. Methods: An analytic, transversal study was made involving 25 healthy volunteers accepted by an informed consent with a normal body mass index, glucose, glycoside hemoglobin, cholesterol and serum triglycerides. The homogeneity of the population was evaluated with anthropometrical and biochemical data using principal component analysis (PCA). The equivalent of $50 \mathrm{~g}$ of carbohydrates test food (tuna) and 50 $g$ of dextrose as food standard was provided for the measure of the glucose curve. The GI was determined by calculating the area under the curve by the triangulation method. The CG was reported as the product of IG by carbohydrate loading provided. Results and Conclusions: The IG of the tuna was $48.01 \pm 17.4$, classified as low, while the CG was $24.0 \pm 8.7$ rated as high. The chemometric analysis by PCA showed that the selection of the normal population for determining the IG, it is important to consider the values of cholesterol and triglycerides.
\end{abstract}

KEY WORDS: Glycemic Index. Glycemic load. Opuntia ficus-indica.

\section{Correspondencia:}

María de Jesús Ibarra-Salas

Av. Dr. Eduardo Aguirre Pequeño, s/n

Col. Mitras Centro

C.P. 64460 , Monterrey, N.L., México

E-mail: maria.ibarras@uanl.mx

Fecha de recepción en version modificada: 06-06-2016

Fecha de aceptación: 08-06-2016

DOI:10.24875/GMM.17002479
Gac Med Mex. 2017;153:473-479

Contents available at PubMed

www.gacetamedicademexico.com 


\section{Introducción}

El concepto del índice glucémico (IG) se desarrolló en 1981 por el Dr. David Jenkins basándose en la hipótesis de Burkitt y Trowell, de 1977, quienes decían que los alimentos que son absorbidos más lentamente tenían beneficios metabólicos en los pacientes con diabetes, problemas cardiovasculares y padecimientos con exceso de adiposidad central con resistencia a la insulina',2.

EI IG es la respuesta glucémica de cierta cantidad de hidratos de carbono disponible en el alimento de prueba comparado con la respuesta glucémica con la misma cantidad de hidratos de carbono de un alimento estándar ${ }^{3}$, como glucosa anhidra o pan blanco consumido por la misma persona. Los alimentos con $I G$ bajo son los que tienen un $I G<55$, con IG moderado tienen 55-70, y con $I G$ alto tienen $>70^{1,3}$. Actualmente no se recomienda usar el IG como único indicador, ya que no contabiliza la cantidad de hidrato de carbono, sino solo la respuesta tras la ingestión ${ }^{1,2}$. El proceso de determinación del IG y de la carga glucémica (CG) es un proceso estandarizado por el Dr. Thomas Wolever ${ }^{4,5}$.

La CG representa el efecto glucémico general de la dieta, porque toma en cuenta tanto el IG como los gramos de hidratos de carbono consumidos en una comida. Se calcula al multiplicar el IG del alimento por los gramos de hidratos de carbono consumidos de dicho alimento y dividiendo entre 100. La CG se clasifica como baja si es $<10$, moderada si es de 1120 y alta cuando es $>20^{1,2}$.

Se sabe que dietas con alimentos con un IG alto promueven la hiperglucemia y la hiperinsulinemia, aumentando a su vez la resistencia de los tejidos periféricos a la insulina y dañando poco a poco las células beta del páncreas, promoviendo así la diabetes mellitus tipo $2^{6}$. Asimismo, afectan el tejido cardiaco por el aumento de la glicación de proteínas, estrés oxidativo y variables hemostáticas; a su vez, disminuye el colesterol ligado a lipoproteínas de baja densidad (LDL), afectando la función endotelial ${ }^{7}$. Estas dietas también se han relacionado con ciertos tipos de cáncer, siendo los más estudiados los de colon y mama ${ }^{8,9}$. Por el contrario, una dieta con un IG bajo se ha visto que induce diversos efectos favorables, tales como una pérdida de peso rápida y un descenso de glucosa e insulina, así como de los triglicéridos ${ }^{7}$. La terapia nutricional más recomendada para el paciente con diabetes mellitus es el uso del IG y la CG para un mejor control de la glucemia y menor comorbilidad ${ }^{1,3}$.

Estudios recientes explican que al desayunar alimentos con un IG bajo se presenta menor sensación de hambre en el transcurso del día; por el contrario, si el desayuno es de IG alto, los mismos sujetos presentan mayor apetito durante el día. Por otro lado, se ha estudiado el efecto en los niños, encontrándose que aquellos que consumen desayunos con IG bajo se han asociado a una mejor memoria espacial y mayor atención durante la mañana ${ }^{10}$.

El consumo de tuna (Opuntia ficus-indica) puede ayudar como parte de la dietoterapia del paciente diabético. Los beneficios están correlacionados con la frecuencia y la cantidad de tuna que se consuma ${ }^{11,12}$.

Según el estudio de Touil, et al. ${ }^{13}$, en 2010 , la tuna está compuesta de un $89 \%$ de agua y un $10.37 \%$ de masa seca, con un $18 \%$ de hidratos de carbono y un $0.17 \%$ de proteínas. Se le atribuyen diferentes propiedades gracias a la cantidad de antioxidantes que contiene. Kuti ${ }^{14}$, en 2004, concluye que la capacidad antioxidante se debe al contenido de flavonoides, ácido ascórbico y contenido de carotenoides ${ }^{15,16}$.

El jugo de tuna tiene un efecto hipoglucemiante y antihiperglucemiante en animales de experimentación con diabetes inducida farmacológicamente, pero no actúa sobre ratas no diabéticas. Esto podría ser porque estimula las células beta del páncreas; además, eleva la hemoglobina en sangre y el colesterol ligado a lipoproteínas de alta densidad (HDL) en ratas diabéticas. También disminuye el colesterol LDL, los triglicéridos y la urea. Un descubrimiento en estas investigaciones fue que el jugo de tuna mejoró el daño pancreático causado por los fármacos inductores de la diabetes en las ratas ${ }^{11,17,18}$.

La Encuesta Nacional de Salud y Nutrición de 2012 reveló una prevalencia combinada de sobrepeso u obesidad en adultos del $73 \%$ para las mujeres y el $69.4 \%$ para los hombres ${ }^{19}$. La tendencia de sobrepeso disminuyó un $5.1 \%$ entre los años 2006 y 2012, mientras que la de obesidad aumentó un $2.9 \%{ }^{19}$.

Más de 371 millones de personas tienen diabetes, y para el año 2030 esta cifra habrá aumentado hasta alcanzar los 552 millones. México ocupa el sexto lugar mundial en el número de personas con diabetes ${ }^{20}$; los Estados con prevalencias más altas de diabetes son Ciudad de México, Nuevo León, Veracruz, Tamaulipas, Durango y San Luis Potosín ${ }^{20}$.

En dos estudios realizados en México se evaluó el IG de varios alimentos de consumo común en México, incluyendo el nopal de la especie Opuntia ficus-indica, 
y se reporta que al ingerirlo solo o con algún otro alimento mantiene un IG bajo, mientras que cereales como arroz, papa, frijol y tortillas de maíz reportan un IG muy similar al pan blanco (alimento estándar). Sin embargo, no se ha encontrado en la literatura ningún reporte de IG del fruto de Opuntia ficus-indica (tuna) $)^{21,22}$.

El IG y la CG son datos importantes como una alternativa en el tratamiento complementario de la diabetes mellitus y de la obesidad. Fue por esto que nos propusimos determinar el IG y la CG del fruto de Opuntia ficus-indica, ya que proviene de una especie vegetal que ha demostrado múltiples beneficios a la salud, además de ser endémico y abundante en nuestro país.

\section{Métodos}

Se realizó un estudio analítico trasversal, el cual se llevó a cabo en el Centro de Investigación en Nutrición y Salud Pública de la Facultad de Salud Pública y Nutrición de la Universidad Autónoma de Nuevo León, Monterrey, N.L., con adultos residentes del mismo Estado. Se incluyeron voluntarios de 18 a 40 años de edad que aceptaron participar en el estudio por medio de consentimiento informado, con un índice de masa corporal (IMC) clasificado como normal (18$24.9 \mathrm{~kg} / \mathrm{m}^{2}$ ) de acuerdo a la Organización Mundial de la Salud ${ }^{23}$, y que negaron enfermedades crónicas no transmisibles, lo cual fue corroborado por medio de estudios bioquímicos que incluyeron biometría hemática completa, química sanguínea y hemoglobina glucosilada ( $\mathrm{HbA1C}$ ). Participaron solo aquellas personas cuyos reportes de laboratorio se encontraron dentro de parámetros normales ${ }^{24}$. Todos los sujetos con estados fisiológicos especiales, como embarazo y lactancia, y las personas con alguna capacidad física diferente que impidiera obtener los parámetros antropométricos, fueron excluidos.

Para analizar las características de la muestra y evidenciar la homogeneidad de los individuos participantes se realizó un análisis de componente principal (PCA) con los datos de los parámetros bioquímicos y antropométricos de los sujetos en estudio por medio del programa Unscrambler versión $9.7^{25}$.

En el análisis bromatológico se determinaron la materia seca y la humedad, las proteínas por el método Kjeldahl con modificación de Winkler, las grasas por el método de goldfish y la fibra cruda conforme a los procedimientos establecidos por la Association of Official Analytical Chemists ${ }^{26}$. Con los resultados obtenidos se calcularon los hidratos de carbono en el alimento de prueba.

Para la determinación del IG y la CG del fruto de Opuntia ficus-indica los sujetos fueron sometidos a una dieta estandarizada durante los días de estudio; la dieta se prescribió de acuerdo a los requerimientos para la edad y el sexo, adecuada en calorías, hidratos de carbono, proteínas y lípidos. Al tercer día se pidió a los participantes que se presentaran con un ayuno nocturno mínimo de 8 horas y se les administró $50 \mathrm{~g}$ de dextrosa anhidra, realizando una curva de glucosa por medio de glucemia en sangre capilar a los minutos $0,15,30,60,90$ y 120; el minuto 0 se consideró cuando el sujeto dio el primer bocado al alimento 0 el primer sorbo de bebida. Dicho procedimiento se repitió al quinto día con el equivalente a $50 \mathrm{~g}$ de hidratos de carbono disponibles en el alimento de prueba (Opuntia ficus-indica). El procedimiento se repitió dos veces.

La determinación del IG se realizó aplicando la técnica de integración del polinomio, con el método desarrollado por el Dr. Wolever, de la Universidad de

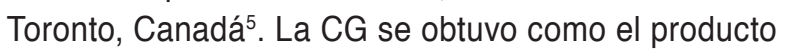
del IG del alimento de prueba y los gramos de hidratos de carbono de la porción dividido entre 100 (Fig. 1).

$$
C G=\frac{(\text { IG del alimento })(\mathrm{g} \text { de HC alimento })}{100}
$$

Figura 1. Fórmula para calcular la carga glucémica de un alimento.

\section{Resultados}

Completaron el estudio 22 sujetos, de los cuales el $86 \%$ eran mujeres y el $13 \%$ eran hombres. La media de edad fue de $24.3 \pm 4.29$ años. El promedio del peso fue de $58.2 \pm 8.5$ kilogramos y el promedio de la talla fue de $1.63 \pm 0.06$ metros, obteniendo un promedio de IMC de $21.7 \pm 1.9 \mathrm{~kg} / \mathrm{m}^{2}$. Estos datos se presentan en la Tabla 1.

Tabla 1. Valores promedio de los indicadores antropométricos de los sujetos de estudio

\begin{tabular}{lccc}
\hline & Rango & Media & DE \\
\hline Edad (años) & $20-40$ & 24.3 & 4.29 \\
Peso $(\mathrm{kg})$ & $43.5-78.2$ & 58.2 & 8.50 \\
Talla $(\mathrm{m})$ & $1.53-1.78$ & 1.63 & 0.06 \\
IMC $\left(\mathrm{kg} / \mathrm{m}^{2}\right)$ & $18.6-24.8$ & 21.7 & 1.90 \\
\hline
\end{tabular}


Se determinaron los datos bioquímicos de glucosa sérica, HbA1c, colesterol y triglicéridos, los cuales se muestran en la Tabla 2. La media de glucosa sérica fue de $84.14 \pm 5.47 \mathrm{mg} / \mathrm{dL}$, el valor promedio de $\mathrm{HbA1c}$ fue de $5.07 \pm 0.253 \%$, el colesterol fue de $173.2 \pm 29 \mathrm{mg} / \mathrm{dL}$ y los triglicéridos mostraron un valor promedio de $77.43 \pm 40 \mathrm{mg} / \mathrm{dL}$.

El PCA con los datos bioquímicos y antropométricos de los sujetos participantes explicó el $97 \%$ de la varianza con dos componentes principales, y se observó que todos los sujetos incluidos en el estudio se distribuyeron en un solo grupo (Fig. 2).

En la Fig. 3 se observa el comportamiento de las variables (loadings), en las que todos los parámetros bioquímicos se encontraron dentro de los valores normales y de igual manera todos contribuyen a la homogeneidad de la muestra. Cabe mencionar que las

Tabla 2. Valores de los indicadores bioquímicos de los sujetos de estudio ${ }^{32}$

\begin{tabular}{lcccc}
\hline & VR & Rango & Media & DE \\
\hline Glucosa $(\mathrm{mg} / \mathrm{dL})$ & $60-110$ & $74-94$ & 84.14 & 5.47 \\
HbA1c $(\%)$ & $4-6$ & $4.6-5.6$ & 5.07 & 0.25 \\
Colesterol $(\mathrm{mg} / \mathrm{dL})$ & $<200$ & $128-232$ & 173.2 & 29.2 \\
Triglicéridos $(\mathrm{mg} / \mathrm{dL})$ & $<150$ & $25-180$ & 77.43 & 40.75
\end{tabular}

DE: desviación estándar; HbA1c: hemoglobina glucosilada; VR: valor de referencia. Fuente: datos obtenidos por el laboratorio clínico.

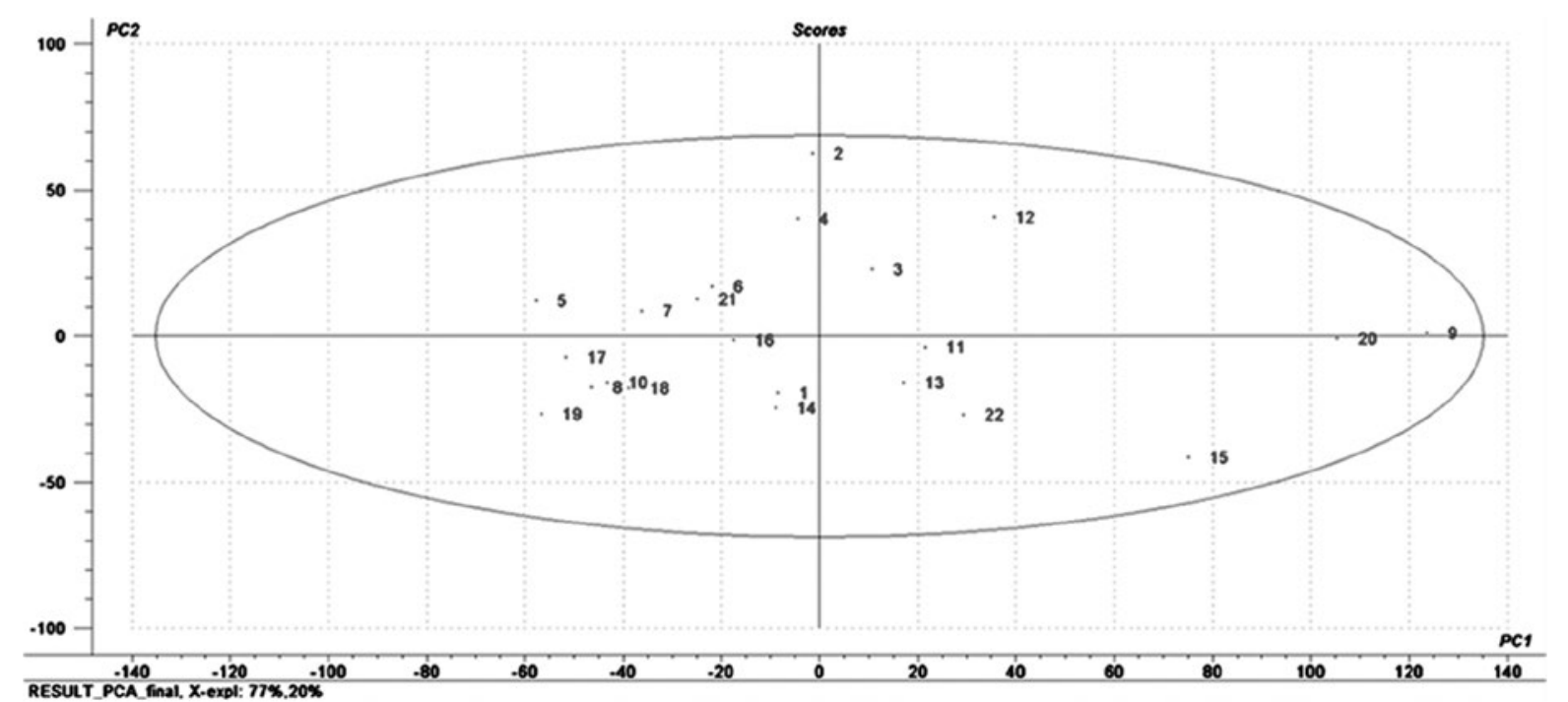

Figura 2. Gráfico de puntos del análisis de componente principal con dos componentes principales y con un $97 \%$ de confianza.

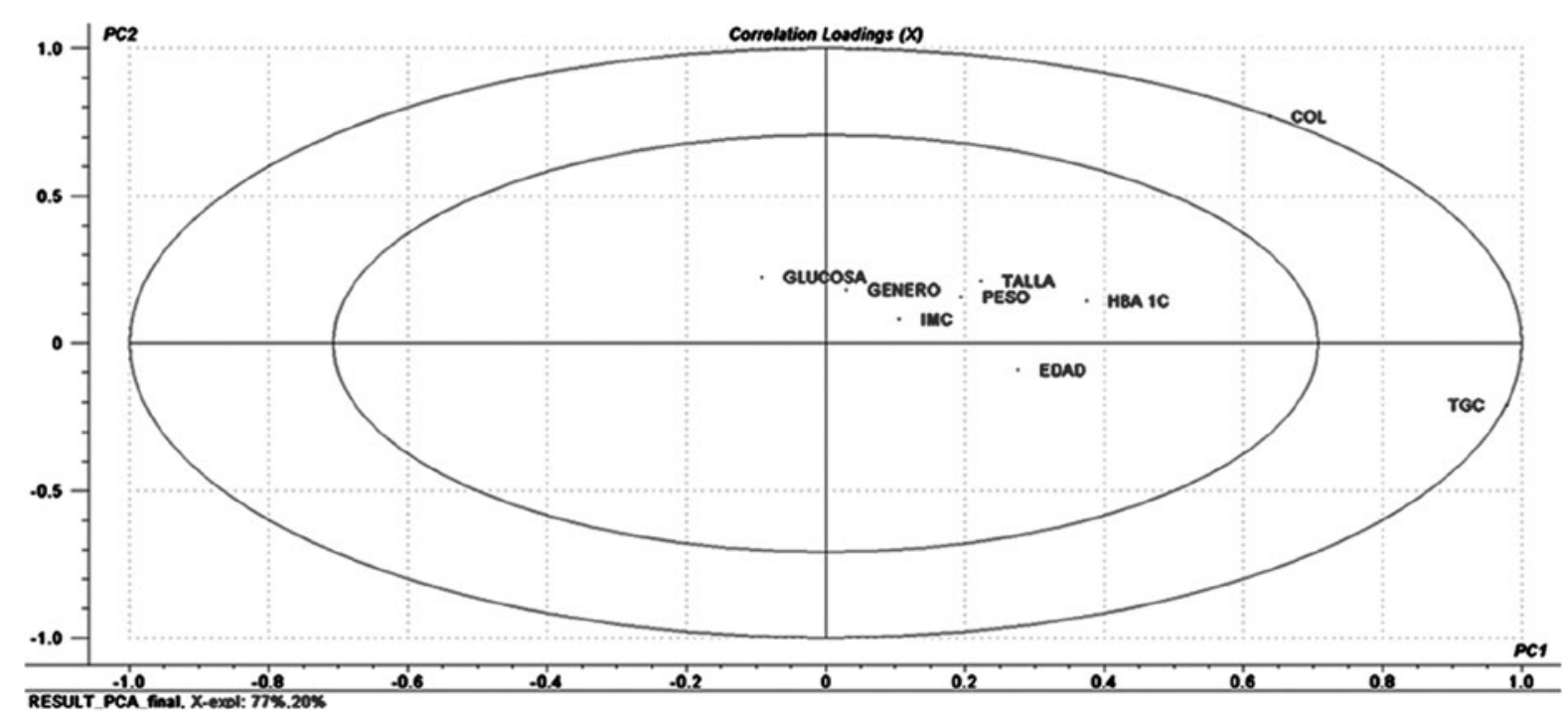

Figura 3. Gráfico de loading de las variables bioquímicas y antropométricas. 
variables colesterol y triglicéridos fueron los parámetros bioquímicos que mayor influencia manifestaron en la dispersión de la muestra; sin embargo, estos se encontraron dentro de valores aceptables (Figs. 2 y 3 ).

En la Tabla 3 se muestran los resultados obtenidos de la composición nutrimental del fruto (tuna) de la especie vegetal Opuntia ficus-indica (los macronutrientes se expresan por $100 \mathrm{~g}$ de alimento).

La cantidad de alimento que proporciona $50 \mathrm{~g}$ de hidratos de carbono fue $387 \mathrm{~g}$ de fruto; cantidad que fue consumida por los sujetos de estudio. Los valores del área bajo la curva, IG y CG del alimento de prueba (tuna), se presentan en la tabla 4.

El IG del fruto de Opuntia ficus-indica fue de 48.01 \pm 17.4 , clasificándose como bajo, y la CG fue de 24 \pm 8.7 , clasificándose como alta.

Se puede observar que el pico máximo de glucemia capilar para el alimento estándar es al minuto 30 , con un promedio de $169 \mathrm{mg} / \mathrm{dL}$, y con el alimento de prueba este pico máximo se presenta en el minuto 15 , con una media de $159 \mathrm{mg} / \mathrm{dL}$. Con el alimento de prueba, la glucemia capilar llega a valores basales en el minuto 90; por el contrario, con el alimento estándar los valores basales de glucemia se alcanzan en el minuto 120 (Fig. 4).

\section{Discusión}

El análisis quimiométrico por PCA demostró que, en la selección de la población normal para la determinación del IG de un alimento, es importante considerar los valores de colesterol y triglicéridos en sangre.

Se logró determinar el IG y la CG del fruto de Opuntia ficus-indica, clasificándolo como bajo y alta, respectivamente.

Se conoce que la tuna tiene diversas propiedades benéficas para la salud, reportadas en diferentes investigaciones en los últimos años. Algunos atributos de dicho fruto son sus efectos antioxidante y antihiperglucemiante; además, las investigaciones más recientes realizadas con jugo y extracto de tuna reportan una regeneración de las células beta pancreáticas in vivo, una disminución de células cancerígenas y la apoptosis de células in vivo de ovario, entre otras ${ }^{17,27-29}$.

En un estudio realizado por Bacardi Gascón, et al. ${ }^{21}$, en 2007, con 36 pacientes con diabetes mellitus tipo 2, se determinó el IG de tres alimentos mexicanos que son consumidos regularmente, como chilaquiles, burritos y quesadillas, y se les adicionó nopal para
Tabla 3. Composición nutricional de la especie vegetal Opuntia ficus-indica (tuna) por 100 gramos

\begin{tabular}{lc}
\hline & Valor por $\mathbf{1 0 0} \mathbf{~ g}$ \\
\hline Energía (kcal) & 56.1 \\
Hidratos de carbono $(\mathrm{g})$ & 12.93 \\
Fibra $(\mathrm{g})$ & 2.6 \\
Proteínas $(\mathrm{g})$ & 0.8 \\
Lípidos $(\mathrm{g})$ & 0.1 \\
Humedad $(\%)$ & 83.2 \\
Cenizas $(\mathrm{g})$ & 0.37 \\
\hline
\end{tabular}

Tabla 4. Valores de índice glucémico y carga glucémica en el alimento de prueba

\begin{tabular}{lcccc}
\hline & Rango & Media & DE & Clasificación \\
\hline Área bajo la curva & - & 141.11 & 54 & - \\
Índice glucémico & $20.67-77.98$ & 48.01 & 17.4 & Bajo \\
Carga glucémica & $10.33-38.99$ & 24.00 & 8.7 & Alta \\
\hline
\end{tabular}

DE: desviación estándar. Fuente: encuesta directa.

comparar el resultado entre el alimento solo y el alimento adicionado con nopal. Se encontró una disminución del IG del 30, el 20 y el 48\%, respectivamente, en los alimentos adicionados con nopal. Se encontró un IG del nopal de 7 y una CG de 35. Los autores atribuyen la acción de la disminución de la glucosa al contenido de fibra soluble, reportando $3 \mathrm{~g}$ presentes en $85 \mathrm{~g}$ de nopal fresco ${ }^{21}$.

En otro estudio que realizaron Frati Munari et al. ${ }^{22}$, en 1991, se estudió el IG de varios alimentos mexicanos comunes en pacientes sanos y en pacientes con diabetes mellitus. Se evaluó el IG de la tortilla de maíz blanco y amarillo, espagueti, arroz, papa, frijol bayo o negro, nopal y cacahuate. De los resultados que se obtuvieron destacan la respuesta glucémica del consumo de nopal en ambos grupos de estudio, ya que no hubo cambio significativo entre la glucosa basal y las posteriores, la elevación máxima de la glucosa después de ingerir nopal fue de 5 a $9 \mathrm{mg} / \mathrm{dL}$ a partir de la cifra al minuto 0 , mientras que los demás alimentos tuvieron un aumento promedio de $10.81 \mathrm{mg} / \mathrm{dL}$ por encima del valor inicial. Reportaron un IG de 15 en el nopal, considerándose bajo ${ }^{22}$. A pesar de la gran cantidad de evidencia científica sobre el fruto estudiado en el aspecto nutricional y fisicoquímico, hasta el momento se desconocían el IG y la CG de la tuna, por lo que era trascendente determinar estos parámetros, ya que en la actualidad es necesario abordar los 


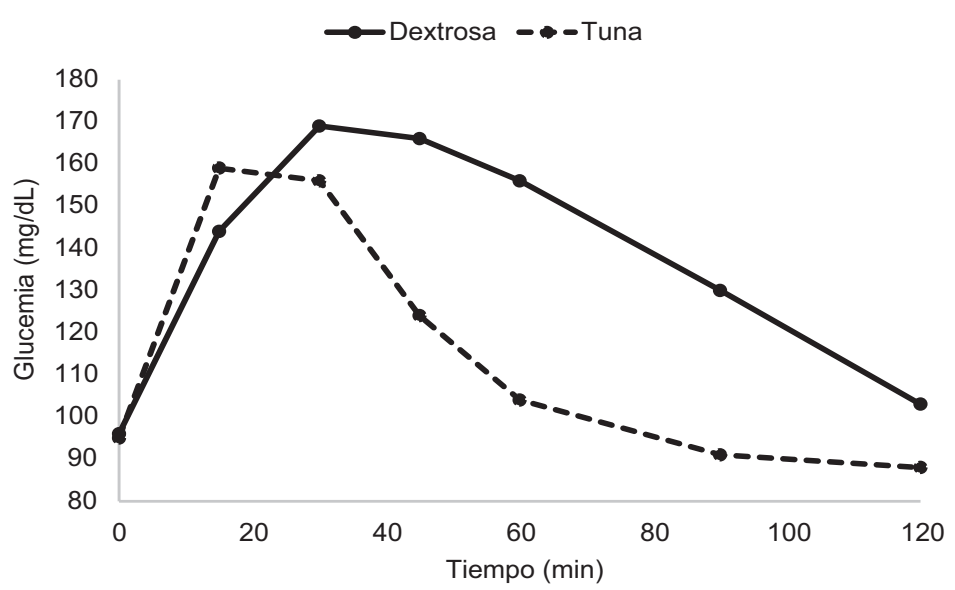

Figura 4. Comportamiento de la glucemia en los diferentes tiempos con el consumo del alimento estándar y del alimento de prueba.

principales problemas de salud, como la obesidad y la diabetes mellitus tipo 2 , con el uso de productos de origen vegetal.

La tuna ha causado gran interés en los últimos años debido a su contenido nutricional. Amaya ${ }^{30}$ reportó en 2009 que la composición del fruto incluye un $85-90 \%$ de humedad, un $0.25-0.44 \%$ de cenizas, un $19 \%$ de hidratos de carbono, un $0.75-5.41 \%$ de proteínas y un $0.12-0.25 \%$ de lípidos. De manera similar, Touil, et al. ${ }^{13}$ encontraron un $89 \%$ de humedad, un $18 \%$ de hidratos de carbono y un $0.17 \%$ de proteínas. En nuestro estudio bromatológico se observó concordancia en cuanto a hidratos de carbono, proteínas, lípidos, humedad y cenizas, pero se encontraron diferencias en el contenido de fibra, ya que este estudio reportó un $2.6 \%$, y Amaya $^{30}$ un $0.02 \%$. Cabe mencionar que, en nuestra investigación, el análisis bromatológico de la tuna se realizó con la variedad cristalina, mientras que los autores citados no especifican la variedad, y tampoco señalan si se consideró el fruto completo o solo la pulpa, ya que estas diferencias podrían modificar el contenido de fibra, así como el IG del alimento ${ }^{13,30}$. En el presente estudio no se realizó un comparativo de la composición nutricional del fruto completo y del fruto sin semilla.

El PCA es una prueba que se aplicó para demostrar que todos los individuos que fueron seleccionados para este estudio reunían las características necesarias para clasificarlos como sanos. Es una prueba estadística que ayuda a visualizar cómo se comportan los grupos de variables ${ }^{25,28,31}$. El análisis se realizó aplicando dos componentes principales que explicaron el $97 \%$ de la varianza. En la Fig. 2 se observa que los sujetos 9 y 20 presentaron mayor dispersión, pero caen dentro del grupo. Al analizar el gráfico de loadings (variables) en la Fig. 2 se pudo identificar que los parámetros bioquímicos que más contribuyen a esta dispersión son el colesterol y los triglicéridos, ya que sus valores de referencia son más amplios. Los resultados de este análisis quimiométrico muestran la importancia de estos dos parámetros bioquímicos en la inclusión de los sujetos en estudio. Cabe mencionar que las variables que más contribuyen a la distribución homogénea son el peso, la talla, el IMC, la HbA1c, la glucosa, el sexo y la edad, tal como se observa en la misma figura.

En este estudio encontramos que el fruto analizado mostró un IG bajo. Se sabe que el IG del alimento varía de acuerdo al tipo de hidratos de carbono, el contenido y el tipo de fibra, las proteínas y la grasa. La determinación se realizó con el fruto rebanado sin cáscara. Estos resultados pueden deberse al contenido de fibra del alimento, ya que se probó el fruto con semillas o por el tipo de hidratos de carbono contenidos en la pulpa. Sin embargo, en el análisis bromatológico no se determinaron los tipos de hidratos de carbono contenidos, siendo esta una limitante de nuestro estudio.

La cantidad de tuna que se administró a los sujetos fue de $387 \mathrm{~g}$, lo cual equivale aproximadamente a seis tunas medianas. La CG obtenida en nuestro estudio fue clasificada como alta. Hay que recordar que la CG depende directamente de la cantidad de hidratos de carbono contenidos en la porción de alimento ingerido y del IG del mismo. Por ello consideramos que, al disminuir la porción del fruto de Opuntia ficus-indica, disminuirá la cantidad de hidratos de carbono que se consumen y por ende se reducirá la CG, por lo que se recomienda ajustar las porciones de este fruto en pacientes con obesidad o diabetes mellitus tipo 2 . 
En cuanto al comportamiento de la glucemia capilar después de la ingesta de $50 \mathrm{~g}$ de hidratos de carbono del fruto, se observó una ligera disminución de la glucemia, ya que el promedio de la glucemia capilar al minuto 0 fue de $96 \mathrm{mg} / \mathrm{dL}$ y al minuto 120 fue de $88 \mathrm{mg} /$ dL. En la literatura no se encontraron estudios que reportaran dichos datos; sin embargo, Abdallah ${ }^{17} \mathrm{y}$ Hassan, et al..$^{18}$, en un estudio en animales de experimentación, mencionan un efecto hipoglucemiante después de la ingesta del jugo de tuna filtrado. De igual manera se recomienda estudiar el efecto hipoglucemiante del fruto posterior a su consumo regular.

\section{Agradecimientos}

Al Dr. Thomas Wolever, de la Universidad de Toronto, Canadá, por su asesoría y apoyo en el desarrollo del proyecto. Al Dr. Rigoberto Vázquez Alvarado, de la Facultad de Agronomía de la UANL, por el apoyo en la identificación y la obtención de la materia prima. Al MSP Jorge Martín Llaca Díaz, del Departamento de Patología Clínica del Hospital Universitario de la UANL, por su apoyo en la realización de los análisis clínicos de los pacientes.

\section{Bibliografía}

1. Casanueva E, Kaufer M, Pérez A, et al. Nutriología médica. México, DF: Editorial Médica Panamericana y Fundación Mexicana para la Salud; 2008.

2. Jenkins D, Kendall C, Agustín L, et al. Glycemic index: overview of implications in health and disease. Am J Clin Nutr. 2002;76(Suppl):266S-73S.

3. Mahan K, Escott-Stump S. Dietoterapia de Krause. $12^{\mathrm{a}}$ ed. Barcelona: Elsevier Masson; 2009

4. Wolever T. The glycemic index. A physiological classification of dietary carbohydrate. Oxford: CABI Publishing; 2006.

5. Wolever T, Jenkins D, Jenkins A, et al. The glycemic index: methodology and clinical implications. J Clin Nutr. 1991;54:846-54.

6. Román L, Guerrero B, García P. Dietoterapia, nutrición clínica y metabolismo. Madrid: Díaz de Santos; 2010.

7. Radulian G, Rusu E, Dragomir A, et al. Metabolic effects of low glycemic index diets. Nutr J. 2009:8:5.

8. Afaghi A, Ziace A, Kiace SM, et al. Glycemic index and glycemic loads of variety of fruits: clinical implementation of fruits serving size in low glycemic load diet. Current Topics in Nutriaceutical Research. 2009;7:157-60.

9. Foster-Powell K, Holt SH, Brand-Miller JC. International table of glycemic index and glycemic load values. Am J Clin Nutr. 2002;76:5-56.

10. Lamport DJ, Hoyle E, Lawton C, et al. Evidence for a second meal cognitive effects in glycemic index evening meals are associate with cognition the collowing morning. Nutr Neurosci. 2011;14:66-71.

11. Castillo GE, Martínez SI. Manual de fitoterapia. Barcelona: Elsevier Masson; 2007.

12. González FMM. Plantas medicinales del noreste de México. Revista IMSS; 1988.

13. Touil A, Chemkhi S, Zagrouba F. Physico-chemical characterization of Opuntia dillenii fruit. International Journal of Food Engineering. 2010;6:1-16.

14. Kuti JO. Antioxidant compounds from four Opuntia cactus pear fruit varieties. Food Chemistry. 2004;85:527-33.

15. Vanaclocha B, Cañigueral S. Fitoterapia: vademecum de prescripción. Barcelona: Elsevier Masson; 2003.

16. Sáenz C, Berger H, Corrales GJ, et al. Utilización agroindustrial del nopal. Boletín de Servicios Agrícolas de la FAO. 2006;162:1-97.

17. Abdallah IZ. Evaluation of hypoglycemic activity of Opuntia dillenii haw fruit juice in streptozotocin-induced diabetic rats. The Egyptian Journal of Hospital Medicine. 2008;33:554-8.

18. Hassan F, El-Razek A, Hassan A. Nutritional value and hypoglycemic effect of prickly cactus pear (Opuntia ficus-indica) fruit juice in alloxan-induced diabetic rats. Australian Journal of Basic and Applied Sciences. 2011;10:356-77.

19. Instituto Nacional de Salud Pública. Encuesta Nacional de Salud Pública y Nutrición 2012. Estado de nutrición, anemia, seguridad alimentaria en la población mexicana.

20. Federación Mexicana de Diabetes. Diabetes en números. 2014. Disponible en: http://fmdiabetes.org/category/diabetes-en-numeros.

21. Bacardi Gascon M, Dueñas Mena D, Jiménez Cruz A. Lowering effect on posprandial glycemic response of nopales added to Mexican breakfasts. Diabetes Care. 2007;30:1264-5.

22. Frati Munari A, Roca Vides R, López Pérez R, et al. Índice glucémico de algunos alimentos comunes en México. Gac Med Mex. 1991;127:163-72.

23. World Health Organization. BMI classification. 2004. Disponible en: http:// apps.who.int/bmi/index.jsp?introPage=intro_3.html

24. Morrison Treseler K. Laboratorio clínico y pruebas de diagnóstico. $3^{\mathrm{a}}$ ed. México: Manual moderno; 1999.

25. CAMO software 2005. The Unscrambler Methods. Disponible en: http:// www.camo.com/downloads/U9.6\%20pdf\%20manual/The\%20Unscrambler\%20Methods.pdf.

26. Horwitz W, Latimer GW. Official methods of analysis of AOAC international. 18th ed. E.E.U.U.: Gaithersburg; 2011.

27. Fernández J, Almela L, Obón J. Determination of antioxidant constituents in cactus fruit. Plant Foods Hum. 2010;65:253-9.

28. Feugang JM, Konarski $P$, Zou D, et al. Nutritional and medicinal use of cactus pear (Opuntia spp.) cladodes and fruits. Front Biosci. 2006;11:2574-89.

29. Liguori G, Di Miceli C, Gugliuzza G, et al. Physiological and technical aspects of cactus pear [Opuntia ficus-indica (L.) Mill.] double rellowering and out-of-season winter fruit cropping. International Journal of Fruit Science. 2006;6:23-34.

30. Amaya-Robles JE. El cultivo de tuna (Opuntia ficus-indica). Gerencia Regional Agraria La Libertad; 2009. p. 35.

31. Miler J, Miller J. Estadística y quimiométrica para química analítica. $4^{\mathrm{a}}$ ed. México: Pearson Prentice Hall; 2002.

32. American Dietetic Association. Clinical practice recommendations. Diabetes Care. 2013;16(Suppl 1):S21. 